This is the post print/version of the article, which has been published in Resuscitation. 2018, 128 , 164-169. http://dx.doi.org/10.1016/j.resuscitation.2018.05.020.

Drom This document has been downloaded from TamPubutafil The Insitutional Repository of University of Tampere

\title{
National early warning score (NEWS) in a Finnish multidisciplinary emergency department and direct vs. late admission to intensive care
}

\author{
Mikko Kivipuro, MB.* Medical School, University of Tampere and Department of Anaesthesia, \\ Tampere University Hospital, PO Box 2000, FI-33521 Tampere, Finland. \\ kivipuro.mikko.m@student.uta.fi
}

Joonas Tirkkonen, MD, PhD.* Department of Intensive Care Medicine and Department of Emergency, Anaesthesia and Pain Medicine, Tampere University Hospital, PO Box 2000, FI-33521 Tampere, Finland. tirkkonen.joonas.o@student.uta.fi

Timo Kontula, MD. Department of Emergency Medicine, Jyväskylä Central Hospital.

Keskussairaalantie 19, FI-40620 Jyväskylä, Finland. tkontula@gmail.com

Juuso Solin, MB. Medical School, University of Tampere and Department of Anaesthesia, Tampere University Hospital, PO Box 2000, FI-33521 Tampere, Finland. solin.juuso.O@student.uta.fi

Jari Kalliomäki, MD. Department of Intensive Care Medicine, Tampere University Hospital, PO Box 2000, FI-33521 Tampere, Finland. jari.kalliomaki@pshp.fi

Satu-Liisa Pauniaho, MD, PhD. Department of Emergency Medicine, Tampere University Hospital, PO Box 2000, FI-33521 Tampere, Finland. satu-liisa.pauniaho@pshp.fi

Heini Huhtala, MSc. Lecturer in Biostatistics, Faculty of Social Sciences, University of Tampere, FI-33014 University of Tampere, Finland. Heini.Huhtala@staff.uta.fi

Arvi Yli-Hankala, MD, PhD. Department of Emergency, Anaesthesia and Pain Medicine, Tampere University Hospital, PO Box 2000, FI-33521 and Faculty of Medicine and Life Sciences, University of Tampere, FI-33014 Tampereen yliopisto, Tampere, Finland. arvi.ylihankala@staff.uta.fi

Sanna Hoppu, MD, PhD. Department of Intensive Care Medicine and Department of Emergency, Anaesthesia and Pain Medicine, Tampere University Hospital, PO Box 2000, FI-33521 Tampere, Finland.sanna.hoppu@pshp.fi

\section{*Equal contribution.}


Corresponding author:

Joonas Tirkkonen, MD, PhD. Department of Intensive Care Medicine and Department of Emergency, Anaesthesia and Pain Medicine, Tampere University Hospital, PO Box 2000, FI-33521 Tampere, Finland. Tel.+358 3311611. tirkkonen.joonas.o@student.uta.fi

Word count abstract: 250

Word count article: 2997

References: 20

Tables and figures: 6 


\section{ABSTRACT}

Objectives: We investigated the national early warning scores (NEWSs) and related outcomes of patients in a tertiary referral center’s multidisciplinary emergency department (ED). Patients were further categorized into three groups: triaged directly to intensive care unit (EDICU), triaged to general ward with later ICU admission (EDwardICU) and triaged to general ward (EDward). NEWSs and subsequent outcomes among these sub groups were compared.

Methods: We conducted a prospective one-month cohort study in Tampere University Hospital's ED, Finland. ED-NEWSs were obtained for all adult patients without treatment limitations, and control (ward) NEWSs were further obtained for the EDwardICU and EDward patients.

Results: Cohort consisted of 1,354 patients with a median ED-NEWS of 2, and higher ED-NEWS was associated with in-hospital mortality (OR 1.26, 95\% CI 1.11-1.42; AUROC 0.75, 0.64-0.86, $\mathrm{p}<0.001$ ) and 30-day mortality (OR 1.27, 1.17-1.39; AUROC 0.78, 0.71-0.84, $<<0.001$ ) irrespective of age and comorbidity. There were 64 patients in EDICU group, 12 patients in EDwardICU group and 1,278 patients in EDward group with median ED-NEWSs of 7, 3 and 2 $(\mathrm{p}<0.001)$, respectively. After the first 24 hours in wards, median NEWSs of the EDwardICU patients had substantially increased as compared with EDward patients (6 vs. 2, $\mathrm{p}<0.001$ ). There were no statistical differences in last NEWS before ICU admission between the EDICU and EDwardICU patients (7 vs. 8, p=0.534), or in ICU severity-of-illness scores or patient outcomes.

Conclusions: ED-NEWS is independently associated with in-hospital and 30-day mortality with acceptable discrimination capability. Direct and late ICU admissions occurred with comparable NEWSs at admission. 
Keywords: National early warning score, emergency department, intensive care 


\section{INTRODUCTION}

During the last decade, various early warning scoring systems have been widely implemented to enable early detection of patient deterioration in prehospital care, on emergency departments and on hospital wards [1-4]. The national early warning score (NEWS) was derived among medical emergency department (ED) patients in the United Kingdom to provide a standardized method for the detection of critically ill patients [5,6]. In December 2017, NEWS received formal endorsement from NHS England to become the early warning system for identifying acutely ill patients in hospitals in England [5]. In ED environments, however, the NEWS has been studied among rather small patient cohorts or pre-selected sub cohorts of patients [6-13]. Further, fixed patient outcomes, such as 30-day mortality, with the adjustment for important confounding factors, has not been studied in a heterogenous ED population [6-13].

NEWS is a continuous variable, and indeed, the trend in NEWS provides objective data on whether a patients' condition is improving or deteriorating [5,8]. Patients admitted to intensive care from general wards have poorer prognosis as compared with those patients admitted directly from EDs and operation rooms $[14,15]$. One of the reasons for this adverse starting point for intensive care may be that the patients in general wards deteriorate hours before adequate interventions take place [15].

In this prospective observational study, we aimed to investigate the adjusted performance of NEWS to predict in-hospital and 30-day mortality among a heterogeneous cohort of patients in a large tertiary referral center's multidisciplinary ED. We further followed the patients admitted to general wards. We then compared the NEWSs and outcomes of the patients subsequently admitted to intensive care with those patients admitted either directly from the ED / never admitted to intensive care. 


\section{METHODS}

\subsection{Ethics}

The Ethics Committee of Tampere University Hospital (Tays) (Approval number R10111; May 5, 2015) approved the study. Written informed consent was waived as no interventions were conducted.

\subsection{Hospital}

Tays is one of the five university hospitals in Finland providing secondary level services for a joint municipal authority of 23 municipalities (population 522,000) and tertiary care for 1.1 million people. Approximately 100,000 patients are treated in hospital's multidisciplinary ED annually (this number includes the primary care patients). The hospital admits 75,000 patients per year (for nonpsychiatric reasons), and 55\% of these patients are non-electively admitted. In 2015 Tays had 538 somatic general ward beds and one mixed surgical-medical intensive care unit (ICU) with 24 beds. Cardiosurgical patients have their own post-operative ICU with 8 beds.

\subsection{Data collection and exclusion criteria}

All patients aged $\geq 18$ who visited the ED, excluding the primary care visits, between June $1^{\text {st }}, 2015$ and July $1^{\text {st }}, 2015$ comprised the initial cohort. The ED nurses collected their vital signs as usual. However, study personnel (= physicians and trained medical students) checked the ED records for missing vital signs required for complete NEWS and supplemented the vital signs measurements if in any way possible. In cases were one to three vital signs were missing despite all efforts, missing values were counted as being within the 'normal' range according to the NEWS; thus a score of zero was used in NEWS calculations with these missing vital signs. Patients with more than three missing vital signs were excluded. Follow-up data on vital signs were similarly collected of those 
patients admitted to hospital's general wards for up to 72 hours; electronic records on vital signs were checked and missing values supplemented as soon as possible. The ED physicians were not aware of the study design or hypothesis. Data related to patient and admission characteristics were collected from the medical records, and data related to ICU admission from the ICU's separate patient management system.

We excluded patients who were under 18 years or who visited a primary care physician. Further exclusion criteria (and the missed patients) are presented in Figure 1. As our study focused on the hospitalized patients, those patients discharged to home or to another health care facility were excluded. Patients who died in ED or who were directly transferred to operation room were further excluded as they either died while receiving immediate interventions or remained in an environment comparable to critical care areas. The final study cohort was further categorized into three groups: triaged directly to intensive care unit (EDICU), triaged to general ward with subsequent ICU admission within 72 hours (EDwardICU) and triaged to general ward (EDward).

\subsection{Outcomes}

The primary outcomes were in-hospital and 30-day mortality. Secondary outcomes for the sub cohort analyses were control NEWSs and SAPS II and APAHCE II scores at ICU admission.

\subsection{Statistical analysis}

No formal power calculations were made, because previous studies had reported statistically significant results regarding the primary outcomes from cohorts of 274-925 patients [7,8,11-13], and we had funding to conduct a one-month study with a rough estimate of 1,500-2,000 patients.

We used the SPSS statistics for Windows, version 23.0, (IBM Corp, Armonk, NY, USA) for statistical analyses. Categorical data are presented as percentages, and continuous data as medians 
and quartiles $\left(\mathrm{Q}_{1}, \mathrm{Q}_{3}\right)$. Differences in baseline characteristics between the groups were tested with the $\chi 2$ test (Fisher's Exact test where appropriate) and the Mann-Whitney U-test. The area under the receiver-operating characteristic (AUROC) curve was used to assess the ability of the ED NEWS to discriminate between the in-hospital and 30-day survivors and non-survivors. We used binary logistic regression analysis with 'enter' method to analyse independent associations with primary outcomes. The Hosmer-Lemeshow test was conducted to present the goodness of-fit of the models. Tests were two-sided; $p<0.05$ was considered significant and 95\% confidence intervals were reported where appropriate. 


\section{RESULTS}

\subsection{Study Population}

A total of 3,311 adult patients visited the ED during the study period, and Figure 1 presents the excluded cases with the final cohort of 1,354 patients. A total of 101 potentially eligible patients (3.1\%) were missed in ED due to logistical reasons. The patients' median age was $65(49,77)$ years, 54\% were male and their median Charlson comorbidity index was 1 (0, 2). Over half of the patients (54\%) were transported in by the emergency medical services (EMS), and 39\% had a surgical reason for the admission. One fifth of the patients (19\%) had a cardiovascular reason for the hospital admission, followed by other medical conditions (16\%), gastrointestinal reasons (13\%), traumas (12\%), respiratory reasons (6.5\%) and other somatic admission reasons (33\%).

Patients' median NEWS in the ED was $2(1,4)$, and ED NEWSs ranged from 0 to 15 . NEWS $\geq 7$ was recorded for $10 \%$ of the patients. All seven vital signs were available for $89 \%$ of the patients; the NEWS was calculated using six vital signs for $8 \%$ of the patients, five vital signs for $2 \%$ and four vital signs for less than $1 \%$ of the patients. The median length of hospital stay for the study population was $3(2,6)$ days, $1.4 \%$ of the patients died during their hospital admission and the 30 day mortality rate was $3.4 \%$.

Sixty-four patients were directly admitted to the ICU from the ED (EDICU), 12 patients were admitted to the ICU within 12-31 hours after initially being admitted to the general wards (EDwardICU), and 1,278 patients were admitted to the general wards and did not require intensive care during their first 72 hours of hospital stay (EDward). The median ED NEWS in the sub groups were 7 (3, 9) (EDICU), 3 (1, 7) (EDwardICU) and 2 (1, 3) (EDward), p<0.001.

\subsection{Mortality and NEWS}


Figure 2 presents how the 30-day mortality increased with higher ED NEWS. Table 1 presents the results of the multivariate logistic regression model. After adjusted for age and CCI, higher ED NEWS was independently associated with in-hospital and 30-day mortality. Figure 3 presents the ROC curves of the ED NEWS for in-hospital and 30-day mortality. The AUROC for NEWS for inhospital mortality was $0.75(0.64-0.86, \mathrm{p}<0.001)$ and $0.78(0.71-0.84, \mathrm{p}<0.001)$ for 30 -day mortality, respectively.

\subsection{Comparison of EDICU patients with EDwardICU patients}

There EDICU and EDwardICU patients were of comparable age and sex, but the patients initially triaged to the general wards had higher CCI and a few chronic illnesses were more prevalent among the EDwardICU patients (Table 2). The median ED NEWS seemed substantially higher among the EDICU patients, but due to the small number of patients in both groups the difference was not statistically significant (Table 2). The ED NEWSs in EDICU group and the last ward NEWSs before the ICU admission in EDwardICU group were comparable. The median SAPS II and APACHE II scores at ICU admission and 30-day mortality all seemed higher among the EDwardICU patients compared with EDwardICU patients, but none of these differences were statistically significant (Table 2).

\subsection{Comparison of EDwardICU patients with EDward patients}

The EDwardICU and EDward patients were of comparable age and sex, but the patients later admitted to the ICU had higher CCI (Table 3). The median ED NEWS was higher among the EDwardICU patients, and their NEWS exceeded the score of six more frequently in the ED. Moreover, NEWSs of the EDwardICU patients had substantially increased during the first 24 hours on general wards, and EDwardICU patients had almost tenfold 30-day mortality as compared with the EDward patients (Table 3). 


\section{DISCUSSION}

\subsection{Key findings}

This is the first study that has investigated the NEWS with adjusted analyses among unselected, DNAR-free patients in a large university hospital's multidisciplinary ED. Our results confirmed that ED NEWS is associated with in-hospital and 30-day mortality irrespective of age and cumulative comorbidity among a very heterogeneous population. The ability of NEWS to discriminate between survivors and non-survivors was acceptable. There were no clinically relevant differences among EDwardICU and EDward patients in the ED, and no statistically significant differences among the EDICU and EDwardICU patients at the time of the ICU admission.

\subsection{Comparison with previous studies}

In 2013 Smith et al. tested the NEWS in a vital signs dataset initially obtained for their previous study investigating the Vital-PAC Early Warning Score, the predecessor of the NEWS $[6,16]$. Although one can question the validation of a slightly modified score (NEWS) in a cohort used to derive its predecessor (ViEWS), with the amount of over 35,000 patients and 199,000 vital signs dataset Smith et al. convincingly showed that NEWS was associated with 24 h morbidity among medical ED patients in univariate analyses [6].

Altogether five studies have investigated the NEWS's ED performance with AUROC analysis $[6,8,9,12,13]$. Smith et al. reported an AUROC of $0.87(0.87-0.88)$ for the combined 24 h outcome of either cardiac arrest, ICU admission or death [6]. De Groot et al. investigated NEWS among 2,280 Dutch ED patients with suspected infection and found that NEWS had 0.67 (0.62-0.72) AUROC for in-hospital mortality, whereas another Dutch study by Alam et al. reported that NEWS had 0.77 (0.62-0.92) AUROC for 30-day mortality among 274 ED patients [8,9]. Keep et al. 
investigated NEWS in an English non-trauma ED with 500 patients, and NEWS had 0.89 (0.840.94) AUROC for severe sepsis [12]. Finally, Sbiti-Rohr et al. reported that among 925 ED patients with community acquired pneumonia, NEWS had an AUROC of 0.65 (0.58-0.72) for 30-day mortality and $0.73(0.67-0.78)$ AUROC for ICU admission [13]. With these results it seems that in study settings the NEWS discriminates patients with worse outcome better the more heterogenous the ED patient cohort is. Our study is in line with this deduction; among our cohort of over 1,300 ED patients from all known somatic specialties the NEWSs ability to discriminate hospital- and 30day survivors and non-survivors was acceptable. This is reassuring, because in clinical work the ED patients are not preselected to specific groups; at the time they are evaluated for the first time by the triage nurses their acute diagnoses are unknown. However, all these previously mentioned studies had indeed very different patient cohorts and outcome measures. The study by Alam et al. is most comparable to ours, and with quite similar results [8]. While we argue that using fixed 30-day mortality as an outcome measure is preferable to short-term outcomes (which, from a patient point of view, is correct), Keep et al. present important data on how NEWS discriminates septic patients with excellent accuracy [12]. With NEWS we do not just want to identify patients with high mortality, we aim to identify 'salvageable' patients before it is too late $[5,17]$.

Adjusting the analyses for relevant co-founders is of utmost importance when the pure chance, randomization, is not utilized. Abott et al. found that higher ED-NEWS was associated with the combined short-term outcome of critical care admission or death within 48 hours after adjusted for age and gender among 431 medical ED patients [7]. Bilben et al. investigated ED-NEWS among 246 dyspneic patients, and higher NEWS was associated with 30-day and 90-day mortality irrespective of age, ASA-score and previously diagnosed COPD [11]. Our current study confirms these findings, as NEWS was associated with 30-day mortality after adjusted for age and cumulative comorbidity among a large, heterogenous and DNAR-free ED patient cohort. Thus, 
higher ED-NEWS is to be taken seriously, whether a patient is young and healthy or old and with several underlying diagnoses.

In 2012 Flabouris et al. found that patients admitted to the ICU directly from the ED had lower APACHE II scores at ICU admission as compared with those patients who were initially admitted to general wards and then subsequently to the ICU [18]. In this large registry study of 43,484 ED patients, $2.3 \%$ of the study population were defined as EDICU patients and $1.1 \%$ as EDwardICU patients, and EDwardICU patients had a 1.5-fold in-hospital mortality as compared with the EDICU patients [18]. One previous, smaller study including 122 patients with either direct or delayed ICU admission reported similarly a 2.5-fold risk for 30-day mortality among the EDwardICU patients [19]. In these studies, the reasons for delayed ICU admissions remained debatable; ED triage categories of EDwardICU patients fell between the EDICU and EDward patients but no clear triage errors were observed $[18,19]$. Our results provide new insight to this matter. Although not a statistically significant difference, EDwardICU patients seemed to have a higher 30-day mortality as in the two previous studies. First, we found that EDwardICU patients had slightly, but statistically significantly more disturbed vital signs according to the NEWS in the ED as compared with the EDward patients. However, median NEWS of two vs. three does not seem a triage error in the ED; the sickest patients with median ED NEWS of seven were directly admitted to the ICU and stabile patients transferred to the general wards, as appropriate. More importantly, during the first 24 hours the vital signs of the EDwardICU patients had substantially worsened as compared with the EDward patients. NEWSs before ICU admission did not differ between the EDwardICU patients and EDICU patients, so according to the vital signs the ICU admissions occurred at comparable state between the sub cohorts. However, the APAHCE II and SAPS II scores (which include laboratory markers etc. and provide a more accurate assessment of a patient's homeostasis as compared with the NEWS) seemed higher among the EDwardICU patients, although this 
difference did not reach statistical difference as in the study by Flabouris et al. [18]. This suggests that long deterioration times on the general wards before appropriate interventions lead to worse overall homeostasis and may explain the worse outcomes among patients with delayed ICU admissions. Patients with disturbed physiology in ED are appropriately admitted to critical care areas without significant delays, whereas in general wards it takes time to act despite vital signs are objectively deteriorating. Unawareness of patients' deteriorating vital signs, and/or insufficient responsiveness to the disturbed vital signs, are common on general wards [20]. Our results emphasize the regular assessment of vital signs in general wards.

Implementation of the NEWS to the prehospital care, ED and general wards would provide an objective continuum to the reassessment of patient's homeostasis through the whole hospital admission [5]. After initial NEWS calculation in prehospital setting [3], reassessment with NEWS in ED provides the first pieces of information of stabilization/ further deterioration. On the other hand, NEWS ED also enables objective follow-up after patients are further admitted to general wards.

\subsection{Limitations}

Firstly, for three percent of the patients, the NEWSs were calculated with only four to five out of seven vital signs. Furthermore, as missing vital signs were supplemented by the study personnel, vital signs were not always measured at the exactly same time points. Secondly, we missed altogether 101 potentially eligible patients; 12 critically ill patients as they were rushed through ED to the ICU, and 89 patients transferred to the general wards. These factors decrease the internal validity of our results. However, these limitations were at comparable level with the previous studies $[8,9,10,11,12]$. Thirdly, although we had a total 1,354 patients in our study, the EDwardICU patient cohort was so small that some statistically significant differences between EDwardICU 
patients and EDICU patients were probably missed due to these sub cohort sizes (type II error).

However, larger registry studies had already documented the differences in mortality rates and we were able to provide vital signs follow-up data for over 1,350 patients $[18,19]$. Fourthly, the external validity of our results is limited by the facts that 1) our ED and hospital characteristics may differ substantially from other institutions and 2) the ICU admission eligibility criteria and the ICU capacity probably vary between hospitals. 


\section{CONCLUSIONS}

Our study confirms that increased NEWS is independently associated with in-hospital and 30-day mortality among patients without treatment limitations in a large University hospital’s multidisciplinary emergency department, irrespective of patients’ age and comorbidities. We were further able to objectively document that NEWSs increased substantially among the patients that were initially triaged to general wards but later admitted to intensive care. These patients seemed to have higher APACHE II scores at ICU admission as compared with the patients who were immediately triaged to intensive care from the ED, although their last NEWSs before ICU admission were comparable.

Conflict of interest statement: Authors declare no conflicts of interests.

Funding: S Hoppu has received a grant (Grant 9S009) from the Competitive Research Funding of the Tampere University Hospital and a grant from the non-profit Paulo Foundation.

Acknowledgements: We would like to acknowledge medical students Verneri Hannula, Pekka Innanen, Erno Iso-aho, Aleksi Lamminen and Miku Tuominen on behalf of the whole study group. 


\section{REFERENCES}

[1] Dundar ZD, Ergin M, Karamercan MA, Ayranci K, Colak T, Tuncar A, et al. Modified Early Warning Score and VitalPac Early Warning Score in geriatric patients admitted to emergency department. Eur J Emerg Med 2016; 23:406-12.

[2] Pullinger R, Wilson S, Way R, Santos M, Wong D, Clifton D, et al. Implementing an electronic observation and early warning score chart in the emergency department: a feasibility study. Eur J Emerg Med 2017;24:e11-6.

[3] Silcock DJ, Corfield AR, Gowens PA, Rooney KD. Validation of the National Early Warning Score in the prehospital setting. Resuscitation 2015;89:31-5.

[4] Tirkkonen J, Olkkola KT, Huhtala H, Tenhunen J, Hoppu S. Medical emergency team activation: performance of conventional dichotomised criteria versus national early warning score. Acta Anaesthesiol Scand 2014;58:411-9.

[5] Royal College of Physicians. National Early Warning Score (NEWS) 2: Standardising the assessment of acute-illness severity in the NHS. Updated report of a working party. London: RCP, 2017.

[6] Smith GB, Prytherch DR, Meredith P, Schmidt PE, Featherstone PI. The ability of the National Early Warning Score (NEWS) to discriminate patients at risk of early cardiac arrest, unanticipated intensive care unit admission, and death. Resuscitation 2013;84:465-70.

[7] Abbott TE, Vaid N, Ip D, Cron N, Wells M, Torrance HD, et al. A single-centre observational cohort study of admission National Early Warning Score (NEWS). Resuscitation 2015;92:89-93. 
[8] Alam N, Vegting IL, Houben E, van Berkel B, Vaughan L, Kramer MH, et al. Exploring the performance of the National Early Warning Score (NEWS) in a European emergency department. Resuscitation 2015;90:111-5.

[9] De Groot B, Stolwijk F, Warmerdam M, Lucke JA, Singh GK, Abbas M, et al. The most commonly used disease severity scores are inappropriate for risk stratification of older emergency department sepsis patients: an observational multi-centre study. Scand J Trauma Resusc Emerg Med 2017;25:91.

[10] Corfield AR, Lees F, Zealley I, Houston G, Dickie S, Ward K, et al. Utility of a single early warning score in patients with sepsis in the emergency department. Emerg Med J 2014;31:482-7.

[11] Bilben B, Grandal L, Søvik S. National Early Warning Score (NEWS) as an emergency department predictor of disease severity and 90-day survival in the acutely dyspneic patient - a prospective observational study. Scand J Trauma Resusc Emerg Med 2016;24:80.

[12] Keep JW, Messmer AS, Sladden R, Burrell N, Pinate R, Tunnicliff M, et al. National early warning score at Emergency Department triage may allow earlier identification of patients with severe sepsis and septic shock: a retrospective observational study. Emerg Med J 2016;33:37-41.

[13] Sbiti-Rohr D, Kutz A, Christ-Crain M, Thomann R, Zimmerli W, Hoess C, et al. The National Early Warning Score (NEWS) for outcome prediction in emergency department patients with community-acquired pneumonia: results from a 6-year prospective cohort study. BMJ Open 2016;6:e011021. 
[14] Goldhill DR, Sumner A. Outcome of intensive care patients in a group of British intensive care units. Crit Care Med 1998;26:1337-45.

[15] Goldhill DR, McNarry AF, Hadjianastassiou VG, Tekkis PP. The longer patients are in hospital before Intensive Care admission the higher their mortality. Intensive Care Med 2004;30:1908-13.

[16] Prytherch D, Smith GB, Schmidt PE, Featherstone PI. ViEWS - towards a national Early Warning Score for detecting adult inpatient deterioration. Resuscitation 2010;81:932_ 7.

[17] Tarassenko L, Clifton DA, Pinsky MR, Hravnak MT, Woods JR, Watkinson PJ. Centile-based early warning scores derived from statistical distributions of vital signs. Resuscitation 2011;82:1013-8.

[18] Flabouris A, Jeyadoss J, Field J, Soulsby T. Direct and delayed admission to an intensive care or high dependency unit following discharge from the emergency department: associated patient characteristics and hospital outcomes. Crit Care Resusc 2012;14:191-7.

[19] Parkhe M, Myles PS, Leach DS, Maclean AV. Outcome of emergency department patients with delayed admission to an intensive care unit. Emerg Med (Fremantle) 2002;14:50-7.

[20] Fuhrmann L, Lippert A, Perner A, Østergaard D. Incidence, staff awareness and mortality of patients at risk on general wards. Resuscitation 2008;77:325-30. 


\section{Legends to the figures}

Figure 1. Emergency department patient flow and the final cohort.

Figure 2. Individual NEWSs and 30-day mortality. A total of nine patients had NEWS $>11$; thus, the last four bars should be interpreted with extreme caution.

Figure 3. The receiver operator characteristics curves (ROC) for the NEWS for in-hospital (upper curve) and 30-day mortality (lower curve). The diagonal reference line presents the ROC of a random predictor, 0.50 . 
Figure 1.

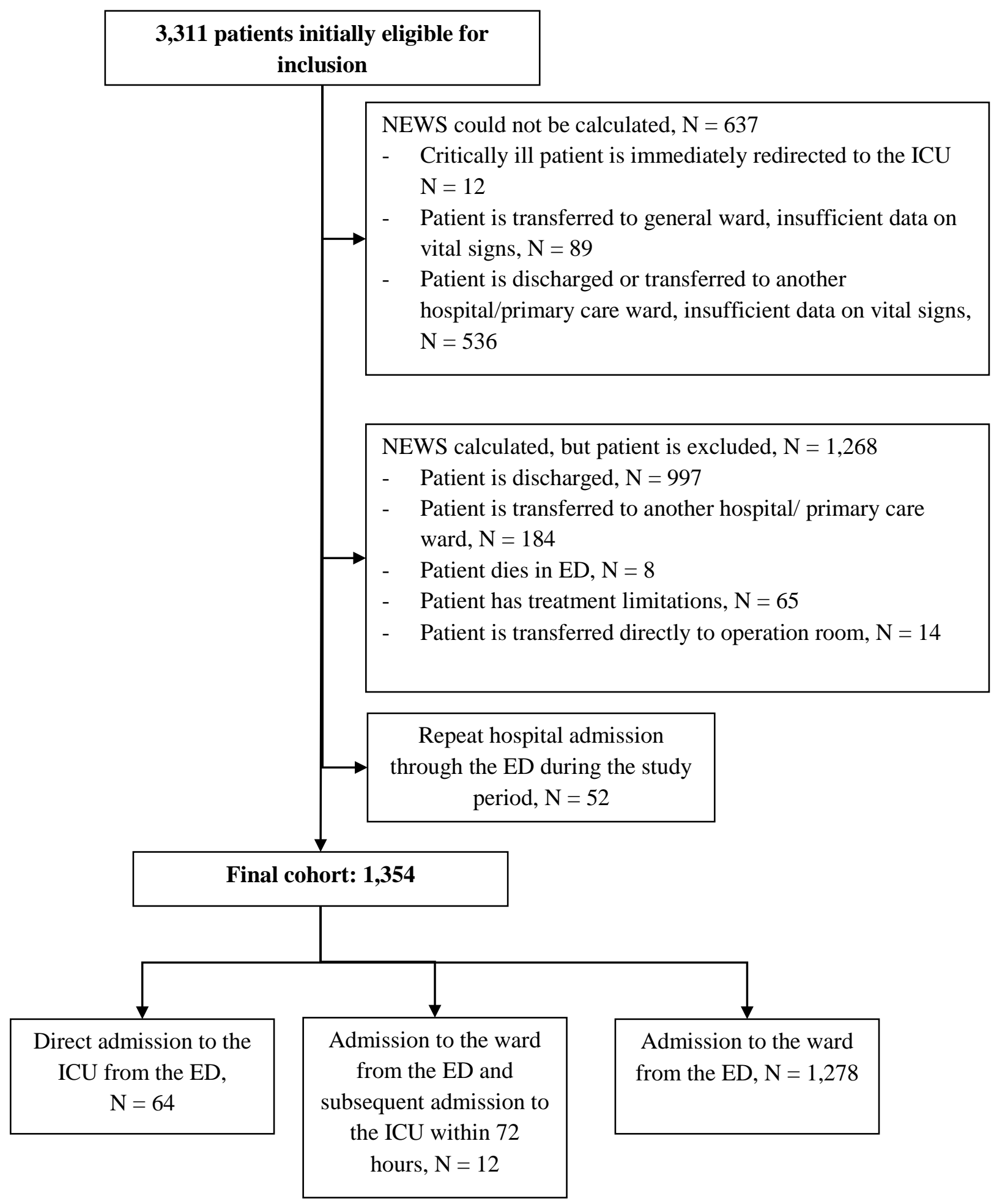


Figure 2.

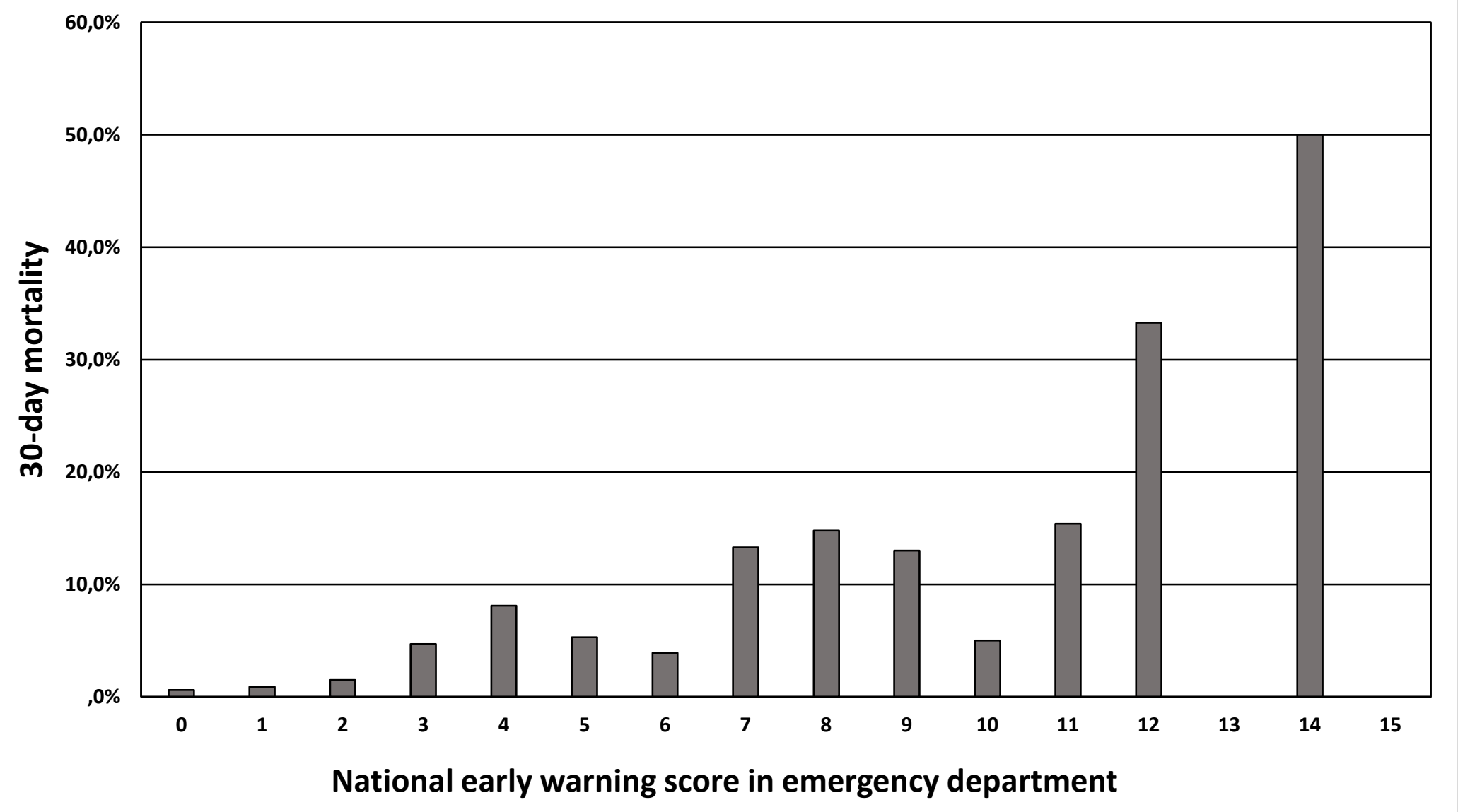


Figure 3.
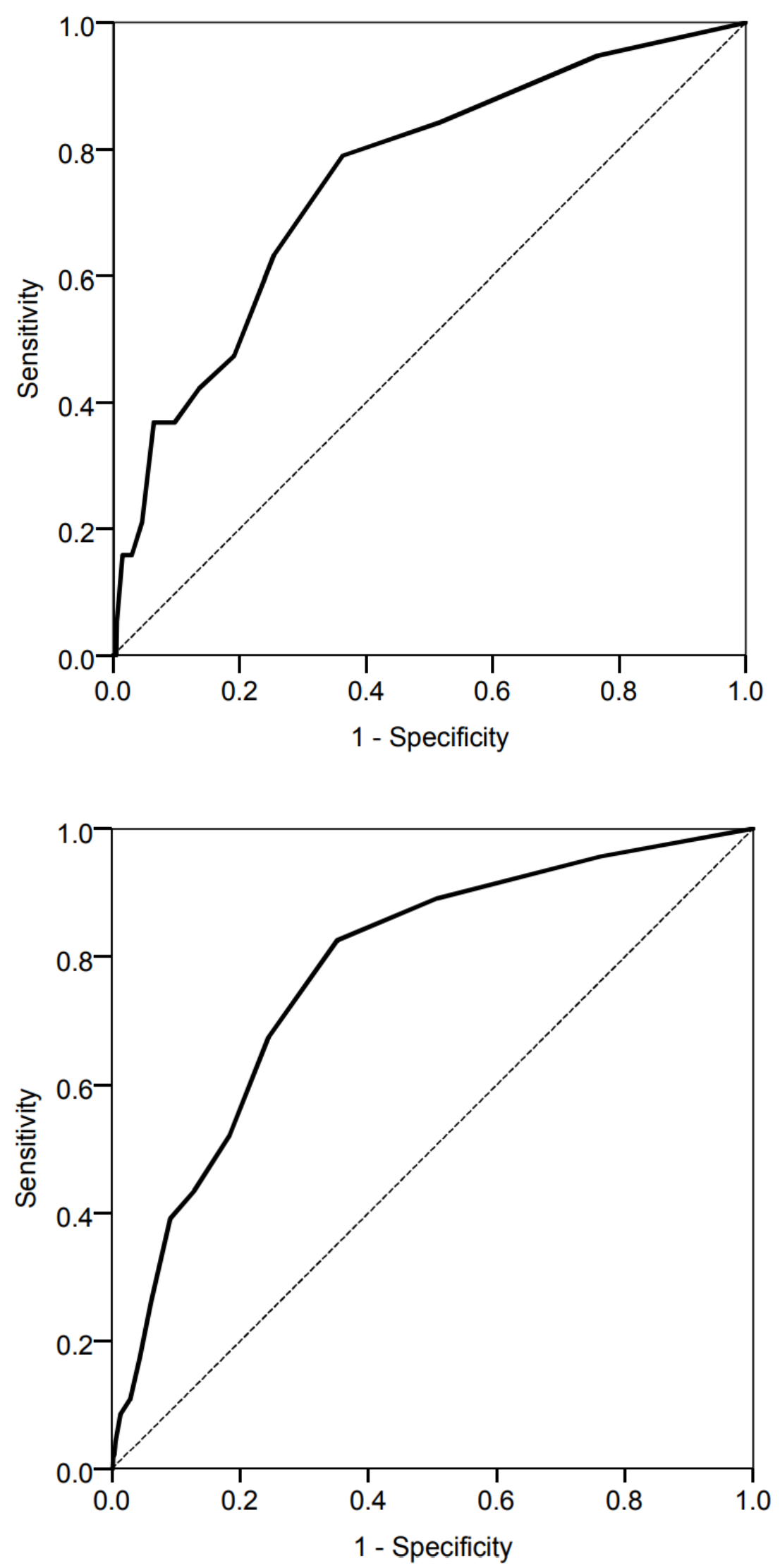
Table 1. Multivariate logistic regression analysis of factors independently associated with inhospital and 30-day mortality, whole cohort $(n=1,354)$.

Multivariate analysis

\begin{tabular}{lccc}
\hline In-hospital mortality (19/1,354) & Odds ratio & $95 \%$ CI & $p$-value \\
\hline Age & 1.02 & $0.99-1.05$ & 0.262 \\
Charlson comorbidity index & 1.24 & $1.02-1.51$ & 0.035 \\
ED NEWS & 1.26 & $1.11-1.42$ & $<0.001$
\end{tabular}

30-day mortality $(46 / 1,354)$

$\begin{aligned} & \text { Age } \\ & \text { Charlson comorbidity index }\end{aligned}$
$\begin{aligned} & 1.02 \\ & \text { ED NEWS }\end{aligned}$
$\begin{aligned} & 1.42 \\ & \text { The Hosmer-Lemeshow goodness-of-fit Chi-squares (4.36, } p=0.823 \text { for the in-hospital mortality }\end{aligned}$
analysis and 6.75, $p=0.563$ for the 30-day mortality analysis) indicated a good fit of the model for
the both the analyses. CI, Confidence interval; ED, emergency department; NEWS, National early
warning score.


Table 2. Characteristics, NEWSs and outcome of EDICU patients compared with the EDwardICU patients.

$$
\text { EDICU }(\mathrm{n}=64) \quad \text { EDwardICU }(\mathrm{n}=\quad p \text {-value }
$$

12)

$\% \quad \%$

Patient characteristics

\begin{tabular}{llll}
\hline Age (median; $\left.\mathrm{Q}_{1}, \mathrm{Q}_{3}\right)$ & $54(37,67)$ & $60(56,68)$ & 0.218 \\
Sex (male) & 66 & 67 & $1.000^{\mathrm{a}}$ \\
Medical patient & 77 & 50 & 0.061 \\
CCI (median; $\left.\mathrm{Q}_{1}, \mathrm{Q}_{3}\right)$ & $1(0,2)$ & $3(1,4)$ & 0.005 \\
Chronic obstructive pulmonary disease & 6.2 & 33 & $0.019^{\mathrm{a}}$ \\
Diabetes with end organ damage & 0.0 & 8.3 & $0.158^{\mathrm{a}}$ \\
Diabetes without end organ damage & 16 & 33 & $0.217^{\mathrm{a}}$ \\
History of myocardial infarction & 6.2 & 8.3 & 1.000 \\
Congestive heart failure & 4.7 & 25 & $0.047^{\mathrm{a}}$ \\
Peripheral arterial disease & 9.4 & 33 & $0.046^{\mathrm{a}}$ \\
Lymphoma & 1.3 & 8.3 & $0.293^{\mathrm{a}}$ \\
Leukaemia & 1.3 & 8.3 & $0.293^{\mathrm{a}}$ \\
Malignant solid tumour & 4.7 & 0.3 & $0.505^{\mathrm{a}}$
\end{tabular}

NEWS

\begin{tabular}{llll}
\hline ED NEWS (median; $\left.\mathrm{Q}_{1}, \mathrm{Q}_{3}\right)$ & $7(3,9)$ & $3(1,7)$ & 0.075 \\
ED NEWS $\geq 7$ & 55 & 33 & $0.217^{\mathrm{a}}$ \\
Last NEWS before ICU admission & $7(3,9)$ & $8(6,9)$ & 0.534
\end{tabular}


Table 3. Characteristics, NEWSs and outcome of EDwardICU patients compared with the EDward patients.

\begin{tabular}{|c|c|c|}
\hline EDwardICU (n = & EDward $(\mathrm{n}=$ & $p$-value \\
\hline 12) & $1,278)$ & \\
\hline
\end{tabular}

Patient characteristics

\begin{tabular}{llll}
\hline Age (median; $\left.\mathrm{Q}_{1}, \mathrm{Q}_{3}\right)$ & $60(56,68)$ & $65(50,77)$ & 0.471 \\
Sex (male) & 67 & 53 & 0.359 \\
Medical patient & 50 & 58 & 0.556 \\
CCI (median; $\left.\mathrm{Q}_{1}, \mathrm{Q}_{3}\right)$ & $3(1,4)$ & $1(0,2)$ & 0.019
\end{tabular}

NEWS

\begin{tabular}{llll}
\hline ED NEWS (median; $\left.\mathrm{Q}_{1}, \mathrm{Q}_{3}\right)$ & $3(1,7)$ & $2(1,3)$ & 0.032 \\
ED NEWS $\geq 7$ & 33 & 7.7 & $0.012^{\mathrm{a}}$ \\
NEWS 24 hours after ward transfer & $6(3,9)$ & $2(0,3)$ & $<0.001$ \\
(median; $\left.\mathrm{Q}_{1}, \mathrm{Q}_{3}\right)$ & & 0.040 \\
Absolute NEWS change first 24 hours & $1(-0.75,6)$ & $0(-1,1)$ & \\
(median; $\left.\mathrm{Q}_{1}, \mathrm{Q}_{3}\right)$ & & &
\end{tabular}

Patient outcome

\begin{tabular}{llll}
\hline Hospital mortality, \% (count) & $8.3(1)$ & $1.1(14)$ & $0.131^{\mathrm{a}}$ \\
30-day mortality, \% (count) & $25(3)$ & $2.7(35)$ & $0.004^{\mathrm{a}}$
\end{tabular}

Data are presented as percentages if not otherwise indicated. ${ }^{a}$ Fisher's Exact test. NEWS, national early warning score; EDwardICU, patients triaged to general ward with subsequent ICU admission; 
EDward, patients triaged to general ward without ICU admission; CCI, Charlson comorbidity index; ED, emergency department. 
(median; $\mathrm{Q}_{1}, \mathrm{Q}_{3}$ )

ICU admission and patient outcome

\begin{tabular}{llcc}
\hline APAHCE II at ICU admission (median; & $19(11,24)$ & $24(18,31)$ & 0.111 \\
$\left.\mathrm{Q}_{1}, \mathrm{Q}_{3}\right)$ & & & \\
SAPS II at ICU admission & $34(20,50)$ & $47(29,57)$ & 0.129 \\
Hospital mortality, \% (count) & $6.2(4)$ & $8.3(1)$ & $1.000^{\mathrm{a}}$ \\
30-day mortality, \% (count) & $13(8)$ & $25(3)$ & $0.365^{\mathrm{a}}$
\end{tabular}

Data are presented as percentages if not otherwise indicated. ${ }^{a}$ Fisher's Exact test. NEWS, national early warning score; EDICU, patients triaged directly to intensive care unit from the emergency department; EDwardICU, patients triaged to general ward with subsequent ICU admission, CCI, Charlson comorbidity index; ICU, intensive care unit; ED, emergency department; APACHE II, Acute Physiology and Chronic Health Evaluation score; SAPS II, Simplified Acute Physiology Score II. 